\title{
Deciphering a subgroup of breast carcinomas with putative progression of grade during carcinogenesis revealed by Comparative Genomic Hybridisation ( $\mathrm{CGH}$ ) and immunohistochemistry
}

\author{
E Korsching', J Packeisen², MW Helms ${ }^{3}$, C Kersting', R Voss ${ }^{4}$, PJ van Diest ${ }^{5}$, B Brandt ${ }^{3}$, E van der Wall ${ }^{6}$, \\ W Boecker' and $\mathbf{H}$ Bürger*,I
}

'Institute of Pathology, University of Münster, Domagkstr. 17, 48149 Münster, Germany; ${ }^{2}$ Institute of Pathology, Klinikum Osnabrück, Domagkstr. 17, 48149 Münster, Germany; ${ }^{3}$ Laboratory Medicine, Institute of Clinical Chemistry, University of Münster, Domagkstr. 17, 48I 49 Münster, Germany; ${ }^{4}$ Institute of Atherosclerosis Research, University of Münster, Domagkstr. 17, 48149 Münster, Germany; ${ }^{5}$ Institute of Pathology, Utrecht University Medical Center, The Netherlands; ${ }^{6}$ Department of Medical Oncology, Utrecht University Medical Center, The Netherlands

\begin{abstract}
Distinct parallel cytogenetic pathways in breast carcinogenesis could be identified in recent years. Nevertheless, it remained unclear as to which tumours may have progressed in grade or which patterns of cytogenetic alteration may define the switch from an in situ towards an invasive lesion. In order to gain more detailed insights into cytogenetic mechanisms of the pathogenesis of breast cancer, the chromosomal imbalances of 206 invasive breast cancer cases were characterised by means of comparative genomic hybridisation $(\mathrm{CGH}) . \mathrm{CGH}$ data were subjected to hierarchical cluster analysis and the results were further compared with immunohistochemical findings on tissue arrays from the same breast cancer cases. The combined analysis of immunohistochemical and cytogenetic data provided evidence that carcinomas with gains of $7 p$, and to a lesser extent losses of $9 q$ and gains of $5 p$, are a distinct subgroup within the spectrum of ductal invasive grade 3 breast carcinomas. These aberrations were associated with a high degree of cytogenetic instability (16.6 alterations per case on average), 16q-losses in over 70\% of these cases, strong oestrogen receptor expression and absence of strong expression of p53, c-erbB2 and Ck 5. These characteristics provide strong support for the hypothesis that these tumours may develop through stages of well- and perhaps intermediately differentiated breast cancers. Our results therefore underline the existence of several parallel and also stepwise progression pathways towards breast cancer.
\end{abstract}

British Journal of Cancer (2004) 90, |422- |428. doi:I0.1038/sj.bjc.660I658 www.bjcancer.com

Published online 2 March 2004

(c) 2004 Cancer Research UK

Keywords: breast cancer; CGH; progression; grade

Technology for the molecular investigation of breast carcinogenesis has evolved tremendously in the last decade with the introduction of whole-genome assays, focusing on RNA (geneexpression analysis) (Alizadeh et al, 2001) and DNA levels within the cell (comparative genomic hybridisation; CGH) (Kallioniemi et al, 1992). In recent years, the use of CGH led to the proposal of an integrated morphological/cytogenetic progression model for breast cancer (Vos et al, 2000; Buerger et al, 2001) with at least two different, parallel cytogenetic pathways: the well-differentiated and the poorly differentiated pathways. The loss of $16 \mathrm{q}$ thereby seems to represent the cytogenetic hallmark of the well-differentiated progression pathway, even though a subset of poorly differentiated tumours also revealed 16q-losses. For these tumours, it remained unclear if they are the end stage of a de-differentiation process via well-differentiated cancers, or if in these cases 16q-losses are merely secondary events. Nevertheless, it also became clear that the majority of breast cancers and its ultimate precursor lesions are characterised by the presence of a distinct genetic alteration

*Correspondence: Dr H Bürger; E-mail: burgerh@uni-muenster.de Received 15 January 2003; revised 16 December 2003; accepted 5 January 2004; published online 2 March 2004
(Buerger et al, 1999a). Our understanding of carcinogenesis in general (Hanahan and Weinberg, 2000) and breast carcinogenesis specifically is challenged by the postulation of differential complex cytogenetic aberration (Buerger et al, 1999b) and RNA expression patterns (Perou et al, 2000). These postulates may enable a better explanation of genotype-phenotype correlations, and may lead to the establishment of new prognostic markers (van't Heer et al, 2002). The obvious increase of knowledge using these techniques is mirrored by the huge amount of data requiring complicated models for biomathematical analysis.

In this study, we would like to show that the re-evaluation of CGHdata by conventional and biomathematical analysis of 206 cases of invasive breast cancer cases provides further insights into cytogenetic events during breast carcinogenesis. These new aspects might close at least some gaps in our current understanding of breast cancer.

\section{MATERIALS AND METHODS}

\section{Material}

A total of 206 invasive breast cancer cases were staged according to the TNM-system. The tumour series represented all subtypes of 
invasive breast cancer, and the tumours were graded according to established protocols (Ellis and Elston, 1998) as G1 $(n=32), \mathrm{G} 2$ $(n=97)$ or $\mathrm{G} 3(n=77)$. The validation of tumour grade has been achieved using consensus panels as previously described (Buerger et al, 2001). In addition, all ductal invasive G3 carcinomas have been subjected to morphometric analysis and the tumour grades revealed significant differences concerning tubule formation, nuclear size, and mitotic rate (Buerger et al, 2001).

\section{Methods}

CGH-analysis The method of CGH-analysis, the corresponding control experiments and the criteria for the evaluation of genetic alterations were performed as previously described (Kallioniemi et al, 1994; Buerger et al, 1999b). CGH was performed on fresh frozen tissue samples.

Biomathematical analysis The CGH ratio profiles and IHC raw data were tabulated in a range from 1 to 2 and from 1 to 3 , respectively. Missing IHC data (9\% of all IHC data) were replaced by the median of that specific score. This procedure approximated the real values in a reasonable manner, and did not bias the evaluation (data not shown here). In the analysis of CGH data combined with IHC data, the scales of both categories were adapted to achieve equally weighted variables. The different lengths of the CGH and IHC data in the feature vector were not changed. To analyse the independent behaviour of the CGH and IHC feature vectors, both categories were also analysed separately. Hierarchical cluster analysis based on a Euclidean distance measure was applied (Alaiya et al, 2002; Harris et al, 2002) with the underlying and tested rationale that our case's set did not include extreme outliers. We used here 'Complete Linkage' as hierarchical cluster method, but we have tested the behaviour of similar methods (above all 'Ward'). The comparative analysis of these further algorithms showed that the basic message in the result sets was the same. Therefore, we decided to choose the common method 'Complete Linkage' because of the comparability to other data. Our evaluations were performed with the statistical platform SPlus6.1-r2 using the functions 'hclust' and 'agnes' based on algorithms as previously described (Kaufman et al, 1999; Struyf et al, 2002). The results are visualised in a dendrogram showing in a graphical way the similarity of the cancer cases. Coloured labels on a branch show the major characteristics of that case.

Flow cytometry DNA ploidy analysis was performed on singlecell suspensions prepared from $50-\mu$ m-thick paraffin samples with a PAS II (Partec Instruments, Arlesheim, Switzerland) mercury lamp-based flow cytometer. DNA histograms were analysed using the MultiCycle (Phoenix Flow Systems, San Diego, CA, USA) cell cycle analysis software according to the previously established protocols (Bergers et al, 1996, 1997).

Tissue microarrays and immunohistochemistry A tissue microarray was constructed according to the standard procedures (Kononen et al, 1998), containing 153 invasive breast cancer cases that were representative of the whole tumour group and were fully characterised by CGH.

Immunohistochemical staining procedures for ER, PR, bcl-2, p21, Ck 5/6, Cyclin D1, Ki-67, p53, Cyclin A, p27 and c-erbB-2 have been performed as previously described. The pretreatment conditions, the source and the dilution of the commercially available primary antibodies, as well as the guidelines for a semiquantitative evaluation have been published elsewhere (Korsching et al, 2002).

In brief, expression was binary graded for ER and PR, bcl-2, p21, Cyclin D1, and Ck 5/6, Ck 8/18 and SMA. Expression was graded from 0 to 3 for Ki-67, p53, Cyclin A and p27 according to the percentage of positive cells. c-erbB-2 was classified according to the Dako-Score (Korsching et al, 2002).

\section{RESULTS}

A detailed description of cytogenetic alteration patterns of the invasive breast cancer cases subjected to biomathematical analysis has been presented before (Buerger and Boecker, 2002). Further detailed characterisation is presented for ductal invasive grade 3 carcinomas only.

DNA flow cytometric and cytogenetic characteristics of ductal invasive grade 3 breast cancer cases with 16q-losses

A total of 15.5 alterations per case were seen in ductal invasive grade 3 breast cancers with 16q-losses. Three out of 12 tumours (25\%) were DNA diploid, one tumour was tetraploid; $66 \%$ were aneuploid. ER and PR were expressed in 70 and $54 \%$ of the cases, respectively. The most frequent chromosomal alterations were 1qgains (84\%), 3q-gains (50\%), 5p-gains (33\%), 7p-gains $(41 \%), 8 \mathrm{p}-$ losses $(64 \%)$, 8q-gains (84\%), 9q-losses (58\%), 11q-losses (50\%), $13 q$-losses $(58 \%), 15 q$-losses (33\%), 17q-gains (33\%) and 20qgains $(50 \%)$.

\section{Biomathematical modelling of cytogenetic alteration patterns in breast cancer}

Biomathematical modelling revealed the presence of multiple clusters in invasive breast cancer as indicated in Figure 1. Distinct clusters included, for example, 1q-gains/16q-lossses, $9 q$-losses $/ 5 p-$ gains/7p-gains/15q-losses or 3q-gains/17q-gains, 20q-gains and $13 q$-losses, respectively, besides a multitude of other clusters (Figure 1).

\section{Immunohistochemical characterisation of all ductal Invasive grade 3 carcinomas/with 16q-losses/with 7p-gains/ with $5 \mathrm{p}$-gains/with $9 \mathrm{q}$-losses}

An overview of the absolute numbers of the respective subgroups and the associated immunohistochemical findings in these subgroups is given in Table 1 . Interestingly, for most of the markers a clear difference of the immuno-profile could be observed for the five different groups. Despite high proliferation indices (Mib-1 and Cyclin A) and the high degree of cytogenetic instability in ductal invasive grade 3 carcinomas with $7 \mathrm{p}$-gains, the frequency of 16q-losses and the percentage of ER/PR + tumours were significantly higher compared to other subgroups. Similar data were obtained by the re-evaluation of ductal invasive grade 3 carcinomas with 5 p-gains or 9q-losses, respectively, even though the percentage, especially of ER and PR + tumours, was lower (Table 1).

\section{DNA flow cytometric and cytogenetic characteristics of ductal invasive grade 3 carcinomas with 7 p-gains}

Since $7 \mathrm{p}$-gains with $16 \mathrm{q}$-losses were among the most frequent alterations, these ductal invasive grade 3 carcinomas were further characterised under this new perspective.

All these tumours were nondiploid, one was DNA tetraploid and the remaining were DNA aneuploid. On average, 16.6 CGH alterations per case were detected.

16q-losses, 9q-losses, 8q-gains and 1q-gains were present in more than $70 \%$ of these tumours. 5 p-gains were detected with a frequency of $52 \%$. In all, $84 \%$ of these tumours were oestrogen receptor positive and $70 \%$ were progesterone receptor positive. None of the tumours revealed a strong c-erbB2 or p53-accumulation. In all, $20 \%$ displayed a $\mathrm{Ck} 5 / 6$ positive phenotype. 


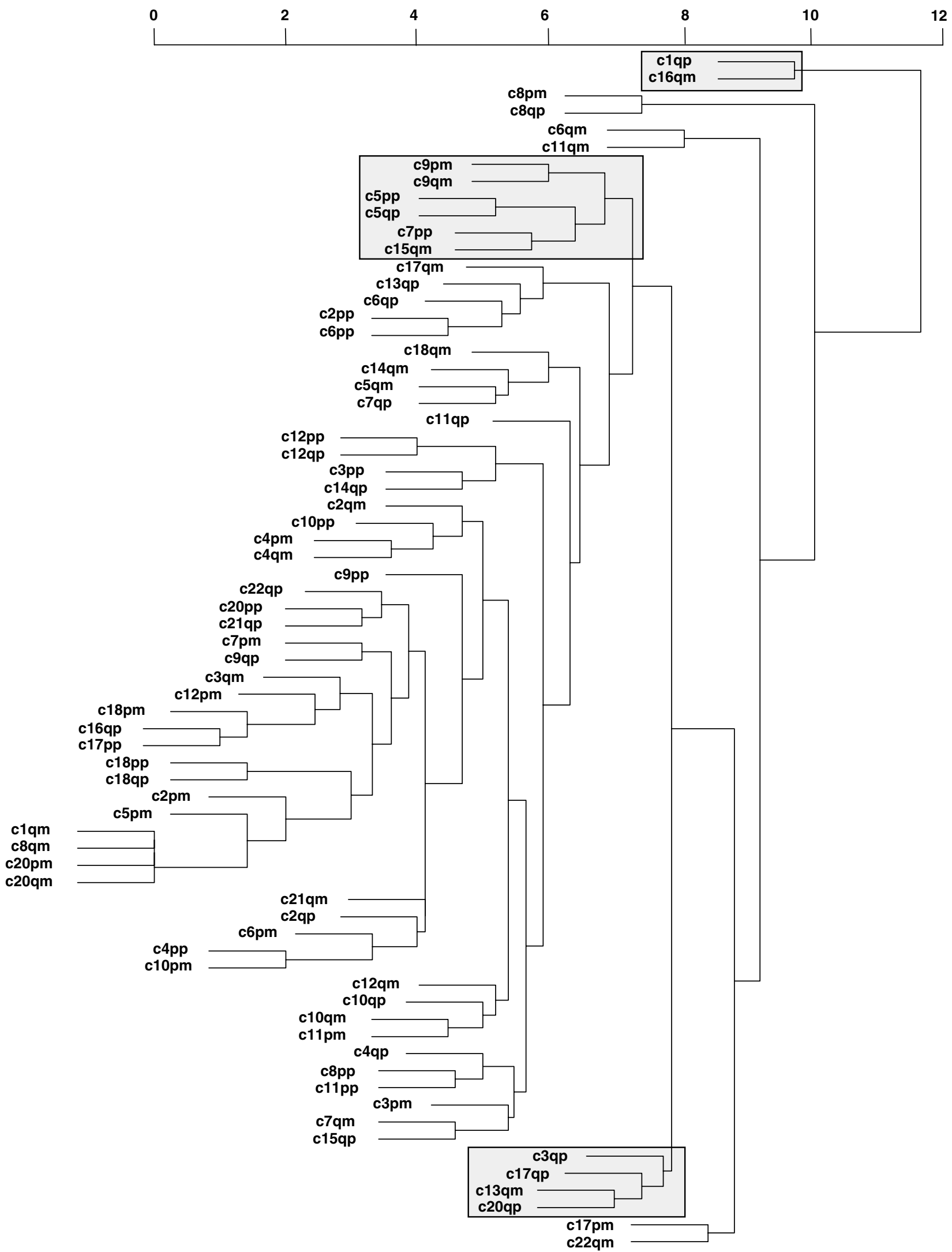

Figure I Dendrogram of cytogenetic alteration patterns revealed from CGH results from 206 invasive breast cancer cases. $p$ and $m$ indicate gains and losses of the short ( $p$ ) and long (q) arm of the respective chromosome. Three clusters of interest are indicated by a frame. Whereas for example the Iqgain/ I 6q-loss cluster is indicative of highly differentiated tumours (Tsuda et al, 1997), two other clusters are indicative of high-grade carcinomas. 


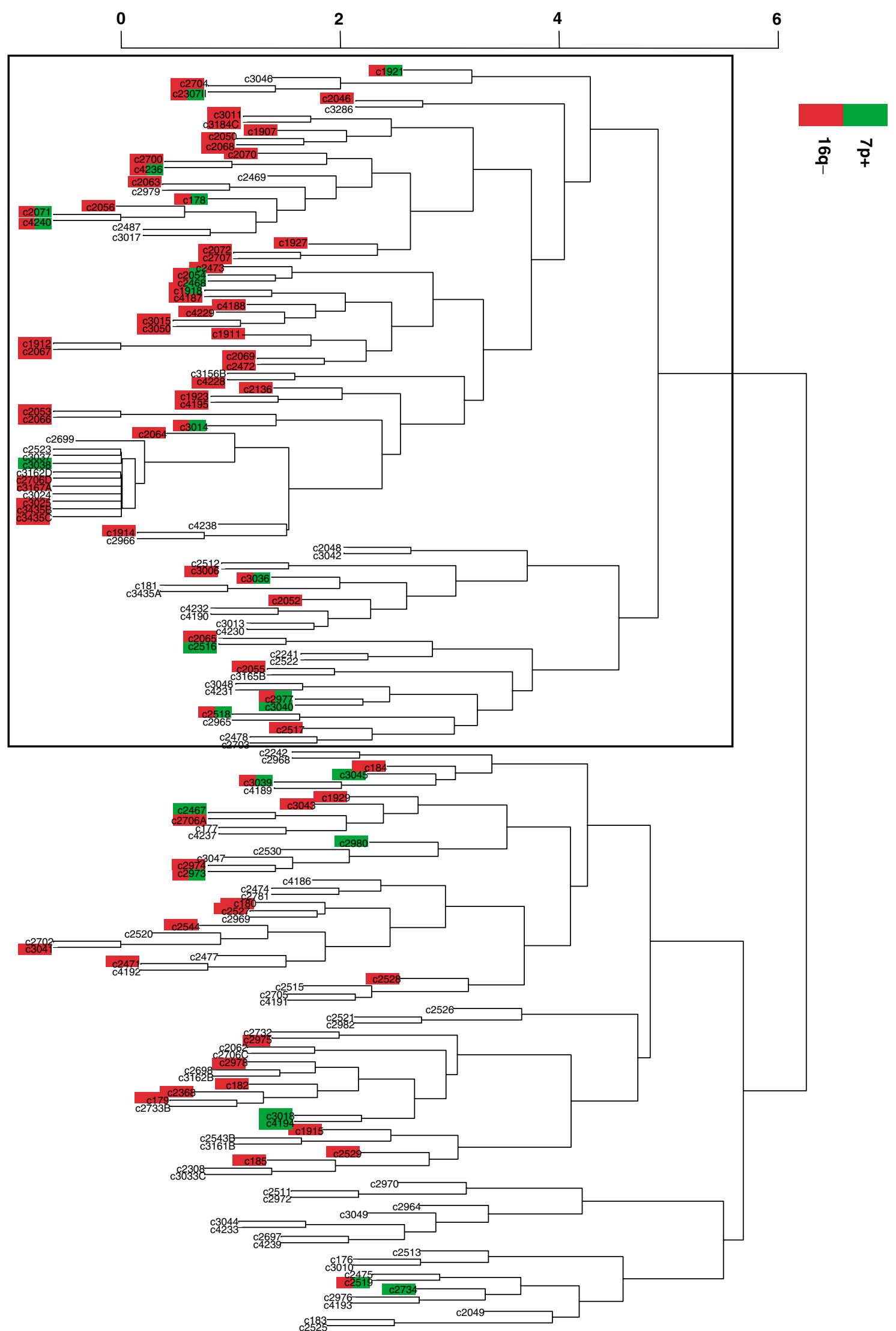

Figure 2 Dendrogram of 153 invasive breast cancer cases, clustered by their protein expression patterns. Two major clusters were formed. One cluster arm (indicated by a frame) is characterised by a significantly higher number of $16 q$-losses and 7p-gains. 16q-losses and 7p-gains are indicated in red and green, respectively. 
Table I Overview of immunohistochemical staining patterns and incidence of $16 q$-losses in subgroups of ductal invasive grade 3 breast cancers

\section{Grade3 all Grade3 with 16q-losses Grade3 with 7p-gains Grade3 with 5p-gains Grade3 with 9q-losses}

Average number of cytogenetic

$\begin{array}{llrr}\text { alterations per case } & 11.7 & 15.5 & 16.6 \\ \text { ER all }(+) \text { cases } & 58 & 70 & 84 \\ \text { PR all }(+) \text { cases } & 63 & 54 & 100 \\ \text { Mib- I }(++)-(+++) & 90 & 90 & 100 \\ \text { c-erbB2 }(-)-(++) & 74 & 80 & 100 \\ \text { p53 }(-)-(+) & 85 & 100 & 40 \\ \text { Cyclin DI all }(+) \text { cases } & 25 & 33 & 60 \\ \text { bcl-2 all }(+) \text { cases } & 24 & 60 & 40 \\ \text { P2I all }(+) \text { cases } & 41 & 50 & 100 \\ \text { p27 }(-)-(++) & 87 & 50 & 20 \\ \text { Cyclin A }(++)-(+++) & 75 & 80 & 7 \\ \text { Ck } 5 \text { all }(+) \text { cases } & 23 & 20 & 100 \\ \text { I6q-losses } & 27 & 100 & \end{array}$

$\begin{array}{rrr}16.6 & 16.8 & 13.0 \\ 84 & & \\ 70 & 75 & 66 \\ 100 & 30 & 58 \\ 100 & 100 & 90 \\ 100 & 80 & 100 \\ 40 & 80 & 81 \\ 60 & 22 & 44 \\ 60 & 44 & 50 \\ 40 & 45 & 40 \\ 100 & 88 & 80 \\ 20 & 90 & 72 \\ 71 & 44 & 20 \\ & 50 & 58\end{array}$

The frequencies are indicated in \%. The average number of cytogenetic alterations per case is given in absolute numbers.

\section{Biomathematical clustering of immunohistochemical expression patterns in invasive breast cancer}

Two major cluster arms could be identified (Figure 2). One of these major cluster arms (indicated by a frame in Figure 1) revealed a lower average number of genetic alterations per case $(7.68 \pm 6.08 \mathrm{vs}$ $8.97 \pm 5.96)$, and a significantly higher frequency of $16 \mathrm{q}$-losses $(P<0.001)$, 7p-gains $(P<0.05)$ and combined $7 \mathrm{p}$-gains $/ 16 \mathrm{q}-$-losses $(P<0.001)$. This cluster contained no tumours with strong $(+++)$ overexpression of c-erbB2, p53 or expression of $\mathrm{Ck} 5 / 6$.

\section{DISCUSSION}

In recent years, cytogenetic progression models for various epithelial neoplasms have been postulated (Jiang et al, 2000; Boecker et al, 2001). However, straightforward statistical interpretation of the complex aberration patterns by conventional analysis of CGH-ratio-profiles provided many limitations. Contemporary bioinformatics procedures can better handle these complex patterns, which may provide a better understanding of mechanisms of carcinogenesis.

In general, the nuclear grade does not seem to change much during tumour progression. Well-differentiated DCIS are associated with invasive carcinomas of low nuclear grade (Holland et al, 1994), while recurrences of breast cancer closely resemble the cytological pattern of their primary (Millis et al, 1998). Despite these general rules, some cases exist with an obvious intratumoral heterogeneity of the nuclear grade, pointing to the possibility of an occasional progression in nuclear grade (Cserni, 2002). Based on our present data on invasive breast cancer cases, we are only able to draw indirect conclusions.

Since this series does not allow a prospective evaluation, the retrospective evaluation of ductal invasive grade 3 carcinomas seemed the most promising approach. This was carried out under the rationale that these tumours, as the extreme end of tumour dedifferentiation, harbour 16q-losses in $20-30 \%$ of cases (Buerger et al, 1999a; Roylance et al, 1999). According to these results, a stepwise evolution via well-differentiated carcinomas could not be excluded theoretically for at least a subgroup of these tumours. From a cytogenetic point of view, these tumours seem to represent the extreme end of tumour de-differentiation with an accumulation of cytogenetic alterations that are rather rare in breast cancers of lower tumour grade. When subjecting the cytogenetic data to a biomathematical approach, alterations mainly seen in ductal invasive grade 3 cancers clearly formed different clusters (Figure 1). The importance of 13q-losses, 17q-gains and 20q-gains

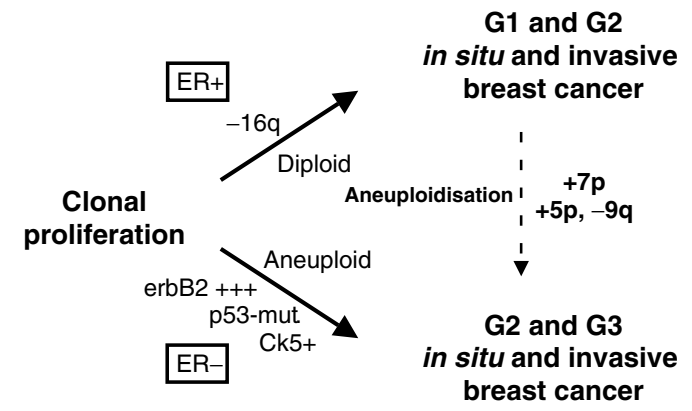

Figure 3 Extended hypothetical model of breast carcinogenesis with evidence for the existence of parallel and stepwise progression pathways. A limited subgroup of well-differentiated breast cancers, characterised by $16 \mathrm{q}$-losses and ER-expression might progress towards poorly differentiated breast cancers, typically not belonging to the c-erbB2 overexpressing, Ck 5/ 6 expressing or 553 accumulating subgroups. This subgroup is characterised by the gain of chromosome 7p, and to a lesser extent by gains and losses of $5 p$ and $9 q$, respectively. Whereas the step of progression is associated with aneuploidisation, the ER-expression remains stable. It will be a focus of further research work to define the frequency of transition between grades.

in breast cancers and their correlation with morphometric and other cytogenetic features have been discussed in detail before (Buerger et al, 2001). No data exist so far with respect to the meaning of $7 \mathrm{p}$ and $5 \mathrm{p}$-gains or $9 \mathrm{q}$-losses in breast cancer, but they are definitely part of far advanced and complex cytogenetic alteration patterns. The immunohistochemical profile of these tumours gave hints that especially $7 \mathrm{p}$-gains are the major hallmark of this alteration pattern. Ductal invasive grade 3 carcinomas with $7 \mathrm{p}$-gains were mainly aneuploid, displayed $16 \mathrm{q}$-losses in $71 \%$ of the cases and displayed a high average number of cytogenetic alterations. Even more important was the oestrogen receptor status. Contrary to expectations for these morphologically faradvanced tumours, $84 \%$ of these tumours were oestrogen receptor positive, $70 \%$ were progesterone receptor positive and none revealed a strong c-erbB2 overexpression or p53 accumulation (hallmarks of gene amplification or mutations, respectively). The whole cytogenetic and immunohistochemical pattern of tumours with $7 \mathrm{p}$-gains, and to a lesser extent $5 \mathrm{p}$-gains as well as $9 \mathrm{q}$-losses (see also cluster in Figure 2), therefore puts them forward as putative examples of poorly differentiated tumours originating from well-differentiated tumours that have acquired a high degree of cytogenetic instability (Figure 3 ). Even though the exact overall 
number of tumours following this pathway cannot be reliably delineated from this approach, this assumption is further supported by the cluster analysis of breast carcinomas based on their immunohistochemical expression pattern. Including a multitude of markers besides ER and PR, similar hints for the meaning of 7 p-gains could be shown. $7 \mathrm{p}$-gains were again predominantly present in a cluster characterised by a low average number of cytogenetic alterations and a significantly higher frequency of $16 \mathrm{q}-$ losses. It is known that distinct chromosomal alteration patterns seem to be associated with distinct protein expression patterns (Isola et al, 1999) and this also seems to hold true for 7p-gains and its associated alterations. The responsible genes involved in this cytogenetic instability associated with 7p-gains are largely unknown. The epidermal growth factor receptor (EGFR), located on $7 \mathrm{p} 12-13$, is an unlikely candidate gene (Briand et al, 1996), since the overexpression of EGFR in invasive breast cancer is associated with oestrogen receptor negativity (Harris et al, 1992). Gene expression analysis studies were able to show that multiple ER-positive breast cancer groups exist, at least one with a very unfavourable prognosis (Perou et al, 2000). Further studies have to show if this specific cytogenetic subgroup with $7 \mathrm{p}$-gains and a high degree of genetic instability represents the cytogenetic homologue for these ER-positive tumours defined by gene expression analysis.
Especially, the finding of a high average number of cytogenetic alterations, shown by itself to be a bad prognostic marker (Isola et al, 1995), and the association with 9q-losses (Gray, 2001) make this likely.

In summary, our results on the one hand show that existing explanation models of breast carcinogenesis are at least partially verified by independent methods, but can also be extended by additional biomathematical procedures. With the introduction of other high-throughput methods such as tissue-arrays (Kononen et al, 1998; Korsching et al, 2002) for the determination of distinct protein expression patterns, or gene expression-profiling (Perou et al, 2000), it will be possible to gain a more complete picture of a single tumour, allowing a more detailed analysis of mechanisms in the pathogenesis of breast cancer.

\section{ACKNOWLEDGEMENTS}

We thank Ulrike Neubert, Petra Meier and Lydia Grote for technical assistance in CGH-analysis, Petra van der Groep for immunohistochemistry, Mark Broeckaert for DNA flow cytometry and Neal Poulin for proofreading the manuscript.

\section{REFERENCES}

Alaiya AA, Franzen B, Hagman A, Dysvik B, Roblick UJ, Becker S, Moberger B, Auer G, Linder S (2002) Molecular classification of borderline ovarian tumours using hierarchical cluster analysis of protein expression profiles. Int J Cancer 98: 895-899

Alizadeh AA, Ross DT, Perou C, van de Rijn M (2001) Towards a novel classification of human malignancies based on gene expression. J Pathol 195: $41-52$

Bergers E, Baak JP, van DP, van GL, Kwee WS, Los J, Peterse HL, Ruitenberg HM, Schapers RF, Somsen JG, van BM, Bellot SM, Fijnheer J (1997) Prognostic implications of different cell cycle analysis models of flow cytometric DNA histograms of 1,301 breast cancer patients: results from the Multicenter Morphometric Mammary Carcinoma Project (MMMCP). Int J Cancer 74: 260-269

Bergers E, Montironi R, van DP, Prete E, Baak JP (1996) Interlaboratory reproducibility of semiautomated cell cycle analysis of flow cytometry DNA-histograms obtained from fresh material of 1,295 breast cancer cases (see comments). Hum Pathol 27: 553-560

Boecker W, Buerger H, Schmitz K, Ellis IO, van Diest PJ, Sinn HP, Geradts J, Poremba C, Herbst H (2001) Ductal epithelial proliferations of the breast: a biological continuum? Comparative genomic hybridization and high-molecular-weight cytokeratin expression patterns. J Pathol 195: $415-421$

Briand P, Nielsen KV, Madsen MW, Petersen OW (1996) Trisomy 7p and malignant transformation of human breast epithelial cells following epidermal growth factor withdrawal. Cancer Res 56: 2039-2044

Buerger H, Boecker W (2002) New insights into the pathogenesis of in situ carcinomas of the breast by means of comparative genomic hybridization. In Ductal Carcinoma of the Breast, Silverstein M (ed) pp 67-76. New York: Lippincott Williams \& Wilkins

Buerger H, Mommers E, Littmann R, Simon R, Diallo R, Poremba C, Dockhorn-Dworniczak B, van Diest PJ, Boecker W (2001) Ductal invasive G2 and G3 carcinomas of the breast are the end stages of at least two different lines of genetic evolution. J Pathol 194: $165-170$

Buerger H, Otterbach F, Simon R, Poremba C, Diallo R, Brinkschmidt C, Dockhorn-Dworniczak B, Boecker W (1999a) Different genetic pathways in the evolution of invasive breast cancer are associated with distinct morphological subtypes. J Pathol 189: $521-526$

Buerger H, Otterbach F, Simon R, Poremba C, Diallo R, Decker T, Riethdorf L, Brinkschmidt C, Dockhorn-Dworniczak B, Boecker W (1999b) Comparative genomic hybridization of ductal carcinoma in situ of the breast - evidence of multiple genetic pathways. J Pathol 187: 396-402

Cserni G (2002) Tumour histological grade may progress between primary and recurrent invasive mammary carcinoma. J Clin Pathol 55: 293-297
Ellis IO, Elston CW (1998) Tumours of the breast. In Diagnostic Histopathology of Tumours, Fletcher CDM (ed) pp 635-689. New York: Churchill Livingstone

Gray J (2001) Quantitative analysis of chromosomal CGH in human breast tumours associates copy number abnormalities with p53 status and patient survival. Proc Natl Acad Sci USA 98: 7952-7957

Hanahan D, Weinberg RA (2000) The hallmarks of cancer. Cell 100: $57-70$

Harris AL, Nicholson S, Sainsbury R, Wright C, Farndon J (1992) Epidermal growth factor receptor and other oncogenes as prognostic markers. J Natl Cancer Inst Monogr 181-187

Harris RA, Yang A, Stein RC, Lucy K, Brusten L, Herath A, Parekh RD, O’Hare MJ, Neville MA, Page MJ, Zvelebil MJ et al (2002) Cluster analysis of an extensive human breast cancer cell line protein expression map database. Proteomics 2: 212-223

Holland R, Peterse JL, Millis RR, Eusebi V, Faverly D, van-de VM, Zafrani B (1994) Ductal carcinoma in situ: a proposal for a new classification. Semin Diagn Pathol 11: 167-180

Isola J, Chu L, DeVries S, Matsumura K, Chew K, Ljung BM, Waldman FM (1999) Genetic alterations in ERBB2-amplified breast carcinomas. Clin Cancer Res 5: $4140-4145$

Isola JJ, Kallioniemi OP, Chu LW, Fuqua SA, Hilsenbeck SG, Osborne CK, Waldman FM (1995) Genetic aberrations detected by comparative genomic hybridization predict outcome in node-negative breast cancer. Am J Pathol 147: 905-911

Jiang F, Desper R, Papadimitriou CH, Schaffer AA, Kallioniemi OP, Richter J, Sauter G, Mihatsch MJ, Moch H (2000) Construction of evolutionary tree models for renal cell carcinoma from comparative genomic hybridization data. Cancer Res 60: 6503-6509

Kallioniemi A, Kallioniemi OP, Piper J, Tanner M, Stokke T, Chen L, Smith HS, Pinkel D, Gray JW, Waldman FM (1994) Detection and mapping of amplified DNA sequences in breast cancer by comparative genomic hybridization. Proc Natl Acad Sci USA 91: 2156-2160

Kallioniemi A, Kallioniemi OP, Sudar D, Rutovitz D, Gray JW, Waldman F (1992) Comparative genomic hybridization for molecular cytogenetic analysis of solid tumours. Science 258: 818-821

Kaufman L, Hubert M, Rousseeuw PJ (1999) Finding Groups in Data: An Introduction to Cluster Analysis. New York: Wiley

Kononen J, Bubendorf L, Kallioniemi A, Barlund M, Schraml P, Leighton S, Torhorst J, Mihatsch MJ, Sauter G, Kallioniemi OP (1998) Tissue microarrays for high-throughput molecular profiling of tumor specimens (see comments). Nat Med 4: 844-847

Korsching E, Packeisen J, Agelopoulos K, Eisenacher M, Voss R, Isola J, van Diest PJ, Brandt B, Boecker W, Buerger H (2002) Cytogenetic alterations 
and cytokeratin expression patterns in breast cancer - integrating a new model of breast differentiation into cytogenetic pathways of breast carcinogenesis. Lab Invest 82: 1525-1533

Millis RR, Barnes DM, Lampejo OT, Egan MK, Smith P (1998) Tumour grade does not change between primary and recurrent mammary carcinoma. Eur J Cancer 34: 548-553

Perou C, Sorlie T, Eisen M, van de Rijn M, Jeffrey SS, Rees CA, Pollack JR, Ross DT, Johnsen H, Akslen LA, Fluge O, Pergamenschikov A, Williams C, Zhu S, Lonning PE, Borresen DA, Brown PO, Botstein D (2000) Molecular portraits of human breast tumours. Nature 406: 747-752

Roylance R, Gorman P, Harris W, Barnes D, Hanby A, Sheer D (1999) Comparative genomic hybridization of breast tumours stratified by histological grade reveals new insights into the biological progression of breast cancer. Cancer Res 59: 1433-1436
Struyf A, Hubert M, Rousseeuw PJ (2002) Integrating robust clustering techniques in S-PLUS. Comput Stat Data Anal 26: 17-37

Tsuda H, Takarabe T, Susumu N, Inazawa J, Okada S, Hirohashi S (1997) Detection of numerical and structural alterations and fusion of chromosomes 16 and 1 in low-grade papillary breast carcinoma by fluorescence in situ hybridization. Am J Pathol 151: 1027-1034

van't Heer L, Dai H, van de Vijver M, He YD, Hart AA, Mao M, Peterse HL, van der Kooy K, Marton MJ, Witteveen AT, Schreiber GJ, Kerkhoven RM Roberts C, Linsley PS, Bernards R, Friend SH (2002) Gene expression profiling predicts clinical outcome of breast cancer. Nature 415: 530 - 536

Vos CB, ter HN, Rosenberg C, Peterse JL, Cleton JA, Cornelisse CJ, van-de VM (2000) Genetic alterations on chromosome 16 and 17 are important features of ductal carcinoma in situ of the breast and are associated with histologic type. Br J Cancer 81: 1410-1418 